\title{
Bioinspired Cellulose Foam with Excellent Water Wicking and Flux Properties Prepared Using Ice Template
}

\author{
Sitian $\mathrm{Ma}^{1}$, Changjun Liu ${ }^{1}$, Yuan $\mathrm{Xu}^{2}$, Linfeng Wang ${ }^{1}$, Han Wang ${ }^{2}$, \\ Weilin $\mathrm{Xu}^{2}$, Yan Zhuang ${ }^{3 *}$, Hongjun Yang ${ }^{1,2^{*}}$
}

1. College of Materials Science and Engineering, Wuhan Textile University, Wuhan, China, 430200

2. Key Laboratory of Green Processing and Functional New Textile Materials of Ministry of Education, Wuhan Textile University, Wuhan, China, 430200.

3. College of Textile Science and Engineering, Wuhan Textile University, Wuhan, China, 430200 .

Corresponding authors

Yan Zhuang:

Wuhan Textile University

No.1 Yangguang Road, Wuhan City, Hubei Province, China, 430200

Tel \& Fax: +086-027-59367577

Email: 837417232@qq.com

Hongjun Yang:

Wuhan Textile University

No.1 Yangguang Road, Wuhan City, Hubei Province, China, 430200

Tel \& Fax: +086-027-59367690

Email: h_j.yang@yahoo.com 

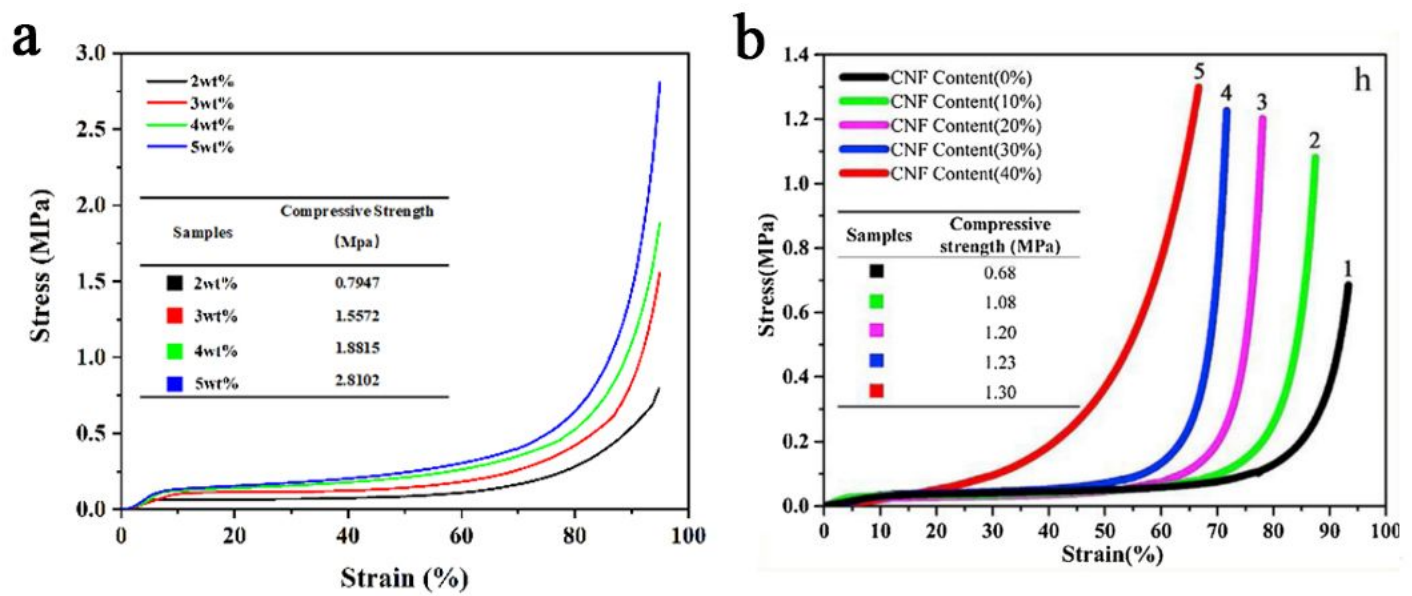

Fig. S1 (a)stress-strain curve of cellulose foam at different concentration in this study; (b) stress-strain curve of cellulose foam in the literature (Ma, S., et al., Lightweight and porous cellulose-based foams with high loadings of zeolitic imidazolate frameworks- 8 for adsorption applications. Carbohydr Polym, 2019. 208: p. 328-335.)

Video S1. Wicking rate test of prepared cellulose membrane.

Video S2. The water flux evaluation of prepared cellulose membrane. 